\title{
Obstetric Cholestasis: A Review and Update
}

\author{
Olawumi Adaramodu and Anthony Kodzo-Grey Venyo
}

\section{ABSTRACT}

Obstetric cholestasis (OC) is a liver disorder that occurs in the late second and early third trimester of pregnancy characterized by pruritus with increased serum bile acids and other liver function tests. The pathophysiology of $\mathrm{OC}$ is still not completely understood. The symptoms and biochemical abnormality rapidly resolve after delivery. OC is associated with an increased risk of adverse obstetrical outcomes. The aetiology of obstetric cholestasis of pregnancy is poorly understood and is thought to be complicated and multifactorial. OC typically occurs in the late second trimester when the oestrogen levels are the highest in pregnancy. The most common complaint is generalized intense pruritus, which usually starts after the 30th week of pregnancy. Pruritus can be more common in the palms and soles and is typically worse at night. Other symptoms of cholestasis, such as nausea, anorexia, fatigue, right upper quadrant pain, dark urine, and pale stool, can be present. Clinical jaundice is rare but may present in $14 \%$ to $25 \%$ of patients after 1 to 4 weeks of the onset of pruritus. Some patients also complain of insomnia as a result of pruritus. Generally, physical examination is unremarkable except for scratch marks on the skin from pruritus. Pruritus is a cardinal symptom of intra-hepatic cholestasis of pregnancy (ICP) and may precede biochemical abnormalities. The diagnosis of intrahepatic cholestasis of pregnancy is via the presence of clinical symptoms pruritus in the third trimester with elevated maternal total serum bile acids and excluding other diagnoses, which can cause similar symptoms and lab abnormalities. Fasting blood samples should be used to check for the total bile salt acid level as it can become elevated in the postprandial state.

Once the diagnosis of $\mathrm{OC}$ of pregnancy is confirmed, immediate treatment is necessary, and the primary goal of therapy is to decrease the risk of perinatal morbidity and mortality and to alleviate maternal symptoms. Maternal pruritus can be alleviated with use of moisturisers and oral antihistamines. Ursodeoxycholic acid (UDCA) is the drug of choice for the treatment of ICP.

Many authors have advocated elective early delivery of women with intrahepatic cholestasis of pregnancy to reduce the risk of sudden foetal death. The Royal College of Obstetricians and Gynaecologists recommends induction of labour after 37+0 weeks of gestation. Obstetric cholestasis of pregnancy is not an indication for Caesarean delivery. Postpartum pruritus typically disappears in the first 2 to 3 days following delivery, and serum bile acid concentrations will normalize eventually. ICP is not a contraindication to breastfeeding, and mothers with a history of ICP in pregnancy can breastfeed their infants. Postpartum monitoring and follow up of bile acids and liver function tests should be done in 4-6 weeks to ensure resolution. Women with the persistent abnormality of liver function test after 6 to 8 weeks require investigation for other aetiologies.

Keywords: Obstetric cholestasis of pregnancy, family history, pruritus, serum bile acid, liver function test, coagulation screen, early induction of labour, vitamin $\mathrm{K}$, postpartum monitoring.
Published Online: December 29, 2020

ISSN: $2736-5476$

DOI: $10.24018 /$ clinicmed.2020.1.1.6

Olawumi Adaramodu *

Salford Primary Care Trust, United

Kingdom.

(e-mail: wumiad@yahoo.com)

Anthony Kodzo-Grey Venyo

North Manchester General Hospital,

United Kingdom.

(e-mail: akodzogrey@yahoo.co.uk)

\section{INTRODUCTION, BACKGROUND, PURPOSE AND SCOPE}

Obstetric cholestasis (OC) is a liver disorder that is associated with pregnancy, and which does manifest predominantly within the third trimester of pregnancy. Obstetric cholestasis of pregnancy is the most common liver disease related to pregnancy [1]. Obstetric cholestasis is also referred to by other terminologies including cholestasis of pregnancy, jaundice of pregnancy, and prurigo gravidarum. Typifying features of $\mathrm{OC}$ include intense pruritus in the absence of a skin rash and abnormal liver function tests (LFT). OC tends to be associated with an increased risk of adverse obstetrical outcomes, which includes stillbirth, 
respiratory distress syndrome, meconium passage, and foetal asphyxiation [2]. The clinical importance of OC lies in the potential foetal risks, spontaneous prematurity, iatrogenic prematurity, intrauterine death, significant maternal morbidity in association with intense pruritus and consequent sleep deprivation. This article contains a review of the literature on $\mathrm{OC}$ with a focus on the clinical presentation, diagnosis, and management of OC.

Aim - to review the literature on obstetric cholestasis.

\section{METHOD}

Internet data bases were searched including Google, Google Scholar, Yahoo, Bing, Pub line and PUBMED. The search words that were used included'; 'intrahepatic, cholestasis'; 'ursodeoxycholic acid', 'sadenosylmethionine', 'vitamin K', 'bile pigments', 'pruritus', 'bilirubin', 'transaminases', 'pregnancy complications', 'dexamethasone', 'congenital', 'neonatal diseases and abnormalities'; 'embryo and foetal development', 'developmental disabilities', 'new born disease', 'prenatal disorder', 'nervous system disorder', 'liver function tests', 'bile acids and salt' and 'aminotransferase'. The National Library for Health and the National Guidelines Clearing House were also searched for relevant guidelines and reviews. Thirty five references were identified that were used to write the article.

\section{RESULTS / REVIEW AND UPDATE OF THE LITERATURE ON OBSTETRIC CHOLESTASIS (OC)}

\section{A. Prevalence}

In England, obstetric cholestasis (also referred to as intrahepatic cholestasis of pregnancy) affects $0.7 \%$ of pregnancies in multi-ethnic populations [3] and 1.2-1.5\% of women of Indian-Asian or Pakistani-Asian origin [4]. Prevalence of $\mathrm{OC}$ is influenced by genetic and environmental factors and does vary between populations globally. For example, in Chile, $2.4 \%$ of all pregnancies are affected by OC, with a prevalence of $5 \%$ in women of Araucanian-Indian origin [5].

\section{B. Diagnosis}

Obstetric cholestasis is a diagnosis of exclusion. Other causes of pruritus and abnormal liver function tests (LFTs) should be excluded. Three steps in making the diagnosis include typical history of pruritus without rash, abnormal Liver Function tests and exclusion of other causes of liver dysfunction.

\section{Causes}

Hormonal, genetic and environmental (e.g., diet, seasonal variations) factors are all likely to be involved. OC may be transmitted in an autosomal dominant manner. Women with OC may be genetically predisposed (one-third of patients have a positive family history) to the cholestatic effect of oestrogens. The supportive evidence for the genetic susceptibility hypothesis lies in the fact that the disease has been observed more in familial clustering patterns, firstdegree relative, and a higher risk of disease recurrence with subsequent pregnancies [6], [7].

\section{Typical History}

Itching is the first symptom and is typically worse at night. This is often widespread and may involve the palms of the hands and the soles of the feet [3]. This latter pattern (soles of feet) is particularly suggestive of OC. When severe, this symptom is intolerable.

If rash is present, then other differential diagnoses need to be considered including polymorphic eruption of pregnancy (PEP) or pemphigoid gestationis [8], [9].

Other features of OC include dark urine, pale stool, steatorrhoea, fatigue, anorexia and jaundice. Clinicians need to enquire about history of cholestasis in previous pregnancies, cholestasis whilst taking oral contraceptive pill, pre-existing liver disease, risk factors for viral hepatitis including travel abroad, drug history and history of allergies or eczema.

\section{E. Abnormal Liver Function Tests}

The upper limit of transaminases, Gamma Glutamyl Transferase (GGT) and bilirubin in pregnancy is $20 \%$ lower than the non-pregnant range [10]. When interpreting the results of the LFT, pregnancy specific reference range should be used. The usual pattern of abnormal LFT is: 2-4 fold rise in transaminases. Alanine transaminase (ALT) is more sensitive. There could be 10-100 fold rise in bile acid levels. Elevated bile acid may be the only biochemical abnormality in a small number of cases with OC [11]-[18]. In some cases of OC, bile acid level could be normal. Other findings that tend to be found in the liver function test results of some patients who have OC include mild elevation in the level of bilirubin, and elevated level of Gamma Glutamyl Transferase (GGT).

\section{F. Investigations to Exclude Other Causes of Liver Disfunction}

Investigations that tend to be undertaken to exclude other causes of liver dysfunction include the following:

Liver and gall bladder ultrasound scan for gall stones, viral screen for hepatitis A, B, C, Epstein Barr and cytomegalovirus, liver autoimmune screen, anti-smooth muscle antibody (Chronic Active hepatitis), antimitochondrial antibody (Primary Biliary Cirrhosis) have been undertaken by some authors [11], [12]. It has been advised that women who have persistent pruritus and normal biochemistry should have LFTs repeated every 2 weeks [19]. It has also been advised that clinicians should be aware of atypical symptoms, which may point to pre-eclampsia or acute fatty liver of pregnancy (AFLP). Clinicians additionally need to be advised that if they are not sure of the diagnosis of OC, they should refer their patients to liver a physician or a gastroenterologist to ensure the correct diagnosis is established.

\section{G. Differentials Diagnoses}

Atypical symptoms can be caused by pre-eclampsia or acute fatty liver of pregnancy (AFLP). If clinicians are in doubt regarding the confirmation of the diagnosis OC then they should refer the patients to liver physicians or gastroenterologists. If a pregnant lady is found to have a rash 
then polymorphic eruption of pregnancy (PEP) or pemphigoid gestationis should also be excluded.

\section{H. Management}

\section{Maternal monitoring:}

Salient points related to the monitoring of pregnant ladies who have OC can be summated to include the ensuing:

- Once OC is diagnosed, LFTs, bile acid and prothrombin time should be measured weekly until delivery.

- Pruritus may precede the derangement of LFT. Therefore, serial (two weekly) LFT and bile acid is advised in women with persistent itching and normal LFTs.

- If LFTs return to normal, obstetric cholestasis is unlikely. Also, if LFTs rise very rapidly consider other differential diagnoses and increase the frequency of monitoring.

- A coagulation screen should be performed.

- Postnatal resolution of symptoms and of biochemical abnormalities is required to secure the diagnosis [11], [12], [14], [15], [17].

\section{Foetal Monitoring}

Stillbirth is the major concern for those involved in the management of obstetric cholestasis. Obstetric Cholestasis has been linked with an increased incidence of passage of meconium, premature delivery, foetal distress, delivery by caesarean section and postpartum haemorrhage.

Recent studies related to perinatal mortality rate from obstetric cholestasis have reported an incidence of 11/1000 (17 foetal or neonatal deaths from all causes in 1538 pregnancies beyond 24 weeks of gestation and live births) [11], [12], [16]-[18], [20]-[22]. However, studies between 2001 and 2011 had reported the perinatal mortality rate of 5.7/1000 (four deaths in 697 pregnancies) [11], [21], [22].

A good guidance towards foetal and maternal monitoring relates to the fact that women who have obstetric cholestasis should be booked in under consultant obstetrician/gynaecologist-led; team based care and the pregnant ladies should give birth within a hospital unit. A number of foetal monitoring techniques have been used to try to predict intrauterine death. These include: maternal detection of foetal movements, growth scan, liquor volume, umbilical artery Doppler scan and cardiotocography. Although reassuring, the usefulness of these tests in the detection of fetal compromise in OC has not been confirmed. Caesarean section rates are high in cases of OC, and these have ranged between $10 \%$ and $36 \%$. Other factors that could contribute to these high rates of caesarean section in obstetric cholestasis include induction of labour, other obstetric indications and of obstetrician or patient anxiety. Despite physiological reasons and a high caesarean section rate, which might suggest an increased risk of postpartum haemorrhage, evidence from current practice does not show this. Postpartum haemorrhage is reported in only five case series, with rates ranging from $2 \%$ to $22 \%$ [11]-[14], [22].

\section{J. Treatment}

General guidelines related to the treatment of pregnant women who are diagnosed as having OC include:

- Pregnant women who are diagnosed as having OC should be involved at all stages of diagnosis and management of the condition. The patient information leaflet on OC should support verbal information.

- Symptomatic control with utilization of emollients such as calamine lotion, aqueous cream with menthol, menthol, diprobase should be undertaken. There are no trial data to support or refute the use of these products. They are safe in pregnancy and clinical experience suggests that for some women they may provide slight temporary relief of pruritus.

- Antihistamines such as chlorphenamine $4 \mathrm{mg}$ 4-6 hourly (up to $16 \mathrm{mg}$ in 24 hours) may provide some welcome sedation at night but do not have a significant impact on pruritus. Activated charcoal [23] and guar gum [24] do not relieve pruritus.

- Ursodeoxycholic acid (UDCA) 8-12 mg per kg daily (1000-1500 mg daily in 2-3 divided doses maximum 2 gm per day) should be discussed and offered to pregnant women who have OC. This does improve pruritus and liver function in women with obstetric cholestasis [25]. The proposed mechanism of action of UCDA is displacement of endogenous bile salts from the bile acid pool. This may enhance bile acid clearance across the placenta from the foetus and may protect the foetal hepatocyte and cardiomyocyte from the toxicity of endogenous bile salts [26], [27].

- If the aforementioned treatment options are insufficient, then Dexamethasone could be used (and this should be decided by the consultant pursuant to discussion with a multidisciplinary management team). Three small observational studies on the use of Dexamethasone, (10 $\mathrm{mg}$ orally for 7 days and then stopping over 3 days) showed improvement in symptoms and biochemistry in some patients[28]-[30]. However, there is general concern about the adverse foetal and neonatal neurological effects with repeated courses of maternally administered Dexamethasone [31].

\section{K. Vitamin K}

Obstetric cholestasis can lead to reduced absorption of dietary fats owing to failure of excretion of bile acids into the gastrointestinal tract and reduced micelle formation. Increased fat excretion in women with obstetric cholestasis has been reported to affect the absorption of fat- soluble vitamins including vitamin $\mathrm{K}$ [32]. Vitamin $\mathrm{K}$ is required for the manufacture of coagulation factors $11, \mathrm{~V} 11$, IX and X. Although the data to support the antenatal use of vitamin $\mathrm{K}$ in obstetric cholestasis are sparse, there are good physiological reasons why this treatment may be beneficial.

Once OC diagnosed, clinicians need to monitor Prothrombin time (PT) and diagnose vitamin K deficiency with prolonged PT. Water soluble vitamin $\mathrm{K}$ tablets (menadiol sodium phosphate) $10 \mathrm{mg}$ orally daily should be prescribed. For women with normal PT, $10 \mathrm{mg}$ menadiol sodium phosphate on alternate days should be given after careful counselling of the patients about the likely benefits but small theoretical risk. Vitamin K deficiency may lead to postpartum haemorrhage, foetal or neonatal bleeding therefore the provision of Vitamin $\mathrm{K}$ should lead to an improved outcome. Use of vitamin $\mathrm{K}$ in higher dose in late pregnancy and labour may increase the risks of neonatal 
haemolytic anaemia, hyper-bilirubinaemia and kernicterus [33].

\section{Timing of Delivery}

The ensuing guidelines to the timing of delivery of pregnant ladies who are diagnosed as having OC would be summated as follows:

- Clinicians should offer women induction of labour after $37+0$ weeks of pregnancy due to risk of stillbirth in higher gestational ages [34].

- The case for intervention at this gestation may be stronger in those with more severe biochemical abnormality.

- Continuous foetal monitoring in labour should be offered including fetal blood sampling and active management of 3rd stage.

- Neonates should receive Vitamin K (Phytomenadione, Konakion neonatal). Benefits and risks of vitamin $\mathrm{K}$ use in OC should be discussed with the woman. Increased risk of postpartum haemorrhage (PPH) is thought to be due to the reduced absorption of vitamin $\mathrm{K}$ dependent coagulation factors secondary to the fat malabsorption in OC.

Current Postpartum haemorrhage rate in OC has been reported as $2-22 \%$, which is comparable to general population. In OC, PPH was more common in those who had not taken vitamin K compared to those who had [11].

\section{Postnatal Care and Follow Up}

The ensuing recommendations would be made relating to the post-natal and follow-up of women with OC following the birth of their children:

- The patients should be followed-up by their General Practitioners (GPs) approximately 6 weeks after delivery to ensure that pruritus resolves and LFTs return to normal.

- $\quad$ LFTs should be repeated in the community one week prior to the planned review by the GP.

- The patients should be reassured about the lack of longterm sequelae for the mother and baby.

- Patients during their follow-up should be told that the risk of recurrence of $\mathrm{OC}$ is high in subsequent pregnancies (45-90\%) [35].

- Utilization of oestrogen containing oral contraceptives should be discouraged as it may precipitate cholestasis.

- The patients in the United Kingdom could be referred to Obstetric Cholestasis support group, which is under the auspices of the British Liver Trust; Obstetric Cholestasis Patient Support Group (http://www.ocsupport.org /index.html). If there are Obstetric Cholestasis Patient Support Groups in other countries, then patients could be referred to the groups. In the United Kingdom, additional patient information leaflets may be obtained from the RCOG and the British Liver Trust.

\section{CONCLUSIONS}

- The management of intrahepatic cholestasis of pregnancy must be a multidisciplinary team approach.
- Typically, whenever feasible, the team should include an obstetrician, maternal-foetal medicine specialist (MFM), gastroenterologist, anaesthetist, and the nursing team.

- A team approach to patient management is vital if one wants to improve outcomes.

\section{REFERENCES}

[1] Clinical Updates in Women's Health Care Summary: Liver Disease: Reproductive Considerations. Obstet Gynecol. 2017 Jan;129(1):236. [PubMed].

[2] Williamson C, Geenes V. Intrahepatic cholestasis of pregnancy. Obstet Gynecol. 2014 Jul; 124 (1):120-33. [PubMed].

[3] Kenyon AP, Tribe RM, Nelson-Piercy C, Girling JC, Williamson C, Seed PT, et al. Pruritus in pregnancy: a study of anatomical distribution and prevalence in relation to the development of obstetric cholestasis. Obstet Med 2010; 3:25-9.

[4] Abedin P, Weaver JB, Egginton E. Intrahepatic cholestasis of pregnancy: prevalence and ethnic distribution. Ethn Health 1999; 4:357.

[5] Reyes H, Gonzalez MC, Ribalta J, Aburto H, Matus C, Schramm G, et al. Prevalence of intrahepatic cholestasis of pregnancy in Chile. Ann Intern Med 1978; 88:487-93.

[6] Turunen K, Helander K, Mattila KJ, Sumanen M. Intrahepatic cholestasis of pregnancy is common among patients' first-degree relatives. Acta Obstet Gynecol Scand. 2013 Sep; 92(9):1108-10. [PubMed].

[7] Pataia V, Dixon PH, Williamson C. Pregnancy and bile acid disorders. Am J Physiol Gastrointest Liver Physiol. 2017 Jul 01; 313(1):G1-G6. [PubMed]

[8] Kenyon AP, Girling JC. Obstetric Cholestasis. In: Studd J, editor Progress in Obstetrics and Gynaecology: Volume 16. Edinburgh: Churchill Livingstone; 2004.p. 37-56.

[9] Ambros-Rudolph CM, Müllegger RR, Vaughan-Jones SA, Kerl H, Black MM .The specific dermatoses of pregnancy revisited and reclassified: results of a retrospective two-centre study on 505 pregnant patients. J Am Acad Dermatol 2006; 54:395-404.

[10] Girling JC, Dow E, Smith JH. Liver function tests in preeclampsia: importance of comparison with a reference range derived for normal pregnancy. Br J Obstet Gynaecol 1997; 104:246-50.

[11] Kenyon AP, Piercy CN, Girling J, Williamson C, Tribe RM, Shennan $\mathrm{AH}$. Obstetric cholestasis, outcome with active management: a series of 70 cases. BJOG 2002; 109: 282-8.

[12] Bacq Y, Sapey T, Bréchot MC, Pierre F, Fignon A, Dubois F. Intrahepatic cholestasis of pregnancy: a French prospective study. Hepatology 1997; 26:358-64.

[13] Reid R, Ivey KJ, Rencoret RH, Storey B. Fetal complications of obstetric cholestasis. Br Med J 1976;1:870-2.

[14] Shaw D, Frohlich J,Wittman BAK, Willms M.A prospective study of 18 patients with cholestasis of pregnancy. Am J Obstet Gynecol 1982; 142:621-5.

[15] Berg B, Helm G, Pertersohn L, Tryding N. Cholestasis of pregnancy. Clinical and laboratory studies.Acta Obstet Gynecol Scand 1986; 65: 107-13.

[16] Fisk NM, Storey GN. Fetal outcome in obstetric cholestasis. Br J Obstet Gynaecol 1988; 95: 1137-43.

[17] Rioseco AJ, Ivankovic MB, Manzur A, Hamed F, Kato SR, Parer JT, et al. Intrahepatic cholestasis of pregnancy: a retrospective casecontrol study of perinatal outcome. Am J Obstet Gynecol 1994; 170:890-5.

[18] Roncaglia N, Arreghini A, Locatelli A, Bellini P, Andreotti C, Ghidini A. Obstetric cholestasis: outcome with active management. Eur J Obstet Gynecol Reprod Biol 2002; 100:167-70.

[19] Kenyon, AP, Piercy CN, Girling J, Williamson C, Tribe RM, Shennan $\mathrm{AH}$. Pruritus may precede abnormal liver function tests in pregnant women with obstetric cholestasis: a longitudinal analysis.BJOG 2001; 108: 1190-2.

[20] Alsulyman OM, Ouzounian JG, Ames-Castro M, Goodwin TM Intrahepatic cholestasis of pregnancy: perinatal outcome associated with expectant management. Am J Obstet Gynecol 1996; 175: 957-60.

[21] Glantz A, Marschall HU, Mattsson LA. Intrahepatic cholestasis of pregnancy: relationships between bile acid levels and fetal complication rates.Hepatology 2004; 40:467-74. 
[22] Lee RH, Kwok KM, Ingles S, Wilson ML, Mullin P, Incerpi M, et al. Pregnancy outcomes during an era of aggressive management for intrahepatic cholestasis of pregnancy. Am J Perinatol 2008; 25: 341-5.

[23] Kaaja RJ, Kontula KK, Räihä A, Laatikainen T. Treatment of cholestasis of pregnancy with peroral activated charcoal.A preliminary study. Scand J Gastroenterol 1994; 2 9:178-81.

[24] Riikonen S, Savonius H, Gylling H, Nikkilä K, Tuomi AM, Miettinen TA. Oral guar gum, a gel-forming dietary fibre relieves pruritus in intrahepatic cholestasis of pregnancy. Acta Obstet Gynecol Scand 2000; 79:260-4.

[25] Jenkins JK, Booth by LA. Treatment of itching associated with intrahepatic cholestasis of pregnancy. Ann Pharmacother 2002; $36: 1462-5$.

[26] Serrano MA, Brites D, Larena MG, Monte MJ, Bravo MP, Oliviera N, et al. Beneficial effect of ursodeoxycholic acid on alterations induced by cholestasis of pregnancy in bile acid transport across the human placenta. J Hepatol 1998; 28:829-39.

[27] Gorelik J, Shevchuk AI,Diakonov I, de Swiet M, Lab M, KorchevY, et al.Dexamethasone and ursodeoxycholic acid protect against the arrhythmogenic effect of taurocholate in an in vitro study of rat cardiomyocytes.BJOG 2003;110:467-74.

[28] Hirvioja M,Tuimala R,Vuori J. The treatment of intrahepatic cholestasis of pregnancy by dexamethasone.Br J Obstet Gynaecol 1992;99:109-11.

[29] Kretowicz E, McIntyre D. Intrahepatic cholestasis of pregnancy, worsening after dexamethasone.Aust N Z J Obstet Gynaecol 1994; 34:211-3.

[30] Diac M,Kenyon A,Nelson-Piercy C,Girling J,Cheng F,Tribe RM, et al. Dexamethasone in the treatment of obstetric cholestasis: a case series. J Obstet Gynaecol 2006; 26:110-4.

[31] Vidaeff AC, Mastrobattista JM. Controversies in the use of antenatal steroids for fetal maturation. Semin Perinatol 2001; 25:385-96.

[32] Reyes H,Radrigan ME,Gonzalez MC, Latorre R,Ribalta J, Segovia N, et al. Steatorrhea in patients with intrahepatic cholestasis of pregnancy.Gastroenterology 1987;93:584-90.

[33] British Medical Association, Pharmaceutical Society of Great Britain.Vitamin K.British National Formulary. London: British Medical Association, Pharmaceutical Society of Great Britain, 2010.

[34] Saleh MM, Abdo KR.Consensus on the management of obstetric cholestasis: national UK survey.BJOG 2007; 114:99-103.

[35] Shaw D, Frohlich J,Wittmann BAK,Willms M.A prospective study of 18 patients with cholestasis of pregnancy. Am J Obstet Gynecol 1982; 142:621-5. 